\title{
Reflection of plane waves at the free surface of a fibre-reinforced elastic half-space
}

\author{
BALJEET SINGH $^{1}$ and SARVA JIT SINGH ${ }^{2}$ \\ ${ }^{1}$ Department of Mathematics, Government College (M), Sector-11, \\ Chandigarh 160 011, India \\ ${ }^{2}$ Department of Mathematics, Maharshi Dayanand University, Rohtak 124 001, \\ India \\ e-mail: baljeet_gill@hotmail.com; s_j_singh@yahoo.com
}

MS received 28 January 2003; revised 14 July 2003

\begin{abstract}
The propagation of plane waves in fibre-reinforced, anisotropic, elastic media is discussed. The expressions for the phase velocity of quasi- $P$ (q $P$ ) and quasi- $S V(\mathrm{q} S V)$ waves propagating in a plane containing the reinforcement direction are obtained as functions of the angle between the propagation and reinforcement directions. Closed form expressions for the amplitude ratios for $\mathrm{q} P$ and $\mathrm{q} S V$ waves reflected at the free surface of a fibre-reinforced, anisotropic, homogeneous, elastic half-space are obtained. These expressions are used to study the variation of amplitude ratios with angle of incidence. It is found that reinforcement has a significant effect on the amplitude ratios and critical angle.
\end{abstract}

Keywords. Anisotropic medium; fibre-reinforced medium; half-space; phase velocity; reflection.

\section{Introduction}

Fibre-reinforced composites are used in a variety of structures due to their low weight and high strength. In most previous investigations on the reflection of waves at the free surface of an elastic half-space, the effect of reinforcement has been neglected. The characteristic property of a reinforced composite is that its components act together as a single anisotropic unit as long as they remain in the elastic condition. Sengupta \& Nath (2001) discussed the problem of surface waves in fibre-reinforced anisotropic elastic media. They expressed the plane strain displacement components in terms of two scalar potentials to decouple the plane motion into q $P$ and q $S V$ waves. Singh (2002) showed that, for wave propagation in fibrereinforced anisotropic media, this decoupling cannot be achieved by the introduction of the displacement potentials. In the present paper, the problem of the reflection of q $P$ and $\mathrm{q} S V$ waves at the free surface of a fibre-reinforced anisotropic elastic half-space is studied by a direct method without the introduction of potentials. Reflection of plane wave at the free surface of an anisotropic half-space has been studied, among others, by Ditri \& Rose (1992), Zilmer et al (1997) and Singh \& Khurana (2002). Hashin \& Rosen (1964) gave the elastic moduli for fibre-reinforced materials. 


\section{Basic equations}

The constitutive equations for a fibre-reinforced linearly elastic anisotropic medium with respect to the reinforcement direction a are (Belfield et al 1983)

$$
\begin{aligned}
\sigma_{i j}= & \lambda e_{k k} \delta_{i j}+2 \mu_{T} e_{i j}+\alpha\left(a_{k} a_{m} e_{k m} \delta_{i j}+e_{k k} a_{i} a_{j}\right) \\
& +2\left(\mu_{L}-\mu_{T}\right)\left(a_{i} a_{k} e_{k j}+a_{j} a_{k} e_{k i}\right)+\beta a_{k} a_{m} e_{k m} a_{i} a_{j},
\end{aligned}
$$

where $\sigma_{i j}$ are the components of stress; $e_{i j}$ are the components of strain; $\lambda, \mu_{T}$ are elastic constants; $\alpha, \beta,\left(\mu_{L}-\mu_{T}\right)$ are reinforcement parameters and $\mathbf{a}=\left(a_{1}, a_{2}, a_{3}\right) ; a_{1}^{2}+a_{2}^{2}+a_{3}^{2}=$ 1 . We choose the fibre-direction as $\mathbf{a}=(1,0,0)$. The strains can be expressed in terms of the displacements $u_{i}$ as

$$
e_{i j}=\frac{1}{2}\left(\frac{\partial u_{i}}{\partial x_{j}}+\frac{\partial u_{j}}{\partial x_{i}}\right) .
$$

For plane strain deformation in the $x_{1} x_{2}$-plane, $\partial / \partial x_{3} \equiv 0, u_{3}=0$. Equation (1) then yields

$$
\begin{aligned}
& \sigma_{11}=A_{11} \frac{\partial u_{1}}{\partial x_{1}}+A_{12} \frac{\partial u_{2}}{\partial x_{2}}, \\
& \sigma_{22}=A_{12} \frac{\partial u_{1}}{\partial x_{1}}+A_{22} \frac{\partial u_{2}}{\partial x_{2}}, \\
& \sigma_{33}=A_{12} \frac{\partial u_{1}}{\partial x_{1}}+\lambda \frac{\partial u_{2}}{\partial x_{2}}, \\
& \sigma_{12}=\mu_{L}\left(\frac{\partial u_{1}}{\partial x_{2}}+\frac{\partial u_{2}}{\partial x_{1}}\right), \\
& \sigma_{31}=\sigma_{32}=0,
\end{aligned}
$$

where

$$
\begin{aligned}
& A_{11}=\lambda+2 \alpha+4 \mu_{L}-2 \mu_{T}+\beta, \\
& A_{12}=\lambda+\alpha, \quad A_{22}=\lambda+2 \mu_{T} .
\end{aligned}
$$

The equations of motion without body forces are

$$
\frac{\partial \sigma_{i j}}{\partial x_{j}}=\rho \frac{\partial^{2} u_{i}}{\partial t^{2}}(i=1,2,3),
$$

using the summation convention. From (2) we note that the third equation of motion in (4) is identically satisfied and the first two equations become

$$
\begin{aligned}
& A_{11} \frac{\partial^{2} u}{\partial x^{2}}+B_{2} \frac{\partial^{2} v}{\partial x \partial y}+B_{1} \frac{\partial^{2} u}{\partial y^{2}}=\rho \frac{\partial^{2} u}{\partial t^{2}}, \\
& A_{22} \frac{\partial^{2} v}{\partial y^{2}}+B_{2} \frac{\partial^{2} u}{\partial x \partial y}+B_{1} \frac{\partial^{2} v}{\partial x^{2}}=\rho \frac{\partial^{2} v}{\partial t^{2}},
\end{aligned}
$$

where we have used the notation $x_{1}=x, x_{2}=y, u_{1}=u, u_{2}=v, B_{1}=\mu_{L}, B_{2}=\alpha+\lambda+\mu_{L}$. 


\section{Propagation of plane waves}

Consider a fibre-reinforced, homogeneous, elastic medium occupying the half-space $y \geq 0$. For plane waves of circular frequency $\omega$, wave number $k$ and phase velocity $c$, propagating in the $x y$-plane and incident at the free boundary $y=0$ at an angle $\theta$ with the $y$-axis (figure 1 ), we may assume

$$
u=U \exp \left(i P_{1}\right), \quad v=V \exp \left(i P_{1}\right),
$$

where $U, V$ are the amplitude factors and,

$$
P_{1}=\omega t-k(x \sin \theta-y \cos \theta),
$$

is the phase factor. For waves reflected at $y=0$, we assume

$$
u=U \exp \left(i P_{2}\right), \quad v=V \exp \left(i P_{2}\right),
$$

where

$$
P_{2}=\omega t-k(x \sin \theta+y \cos \theta)
$$

is the phase factor associated with reflected waves. Making use of (7) or (8) in (5) and (6), we obtain

$$
\begin{aligned}
& -\left(D_{1}-\rho c^{2}\right) U \pm B_{2} \sin \theta \cos \theta V=0, \\
& \pm B_{2} \sin \theta \cos \theta U-\left(D_{2}-\rho c^{2}\right) V=0,
\end{aligned}
$$

where the upper sign corresponds to the incident waves and the lower sign corresponds to the reflected waves. $D_{1}, D_{2}$ are given by

$$
\begin{aligned}
& D_{1}(\theta)=A_{11} \sin ^{2} \theta+B_{1} \cos ^{2} \theta, \\
& D_{2}(\theta)=A_{22} \cos ^{2} \theta+B_{1} \sin ^{2} \theta .
\end{aligned}
$$

Equations (9) and (10) can have a nontrivial solution only if

$$
\left|\begin{array}{cc}
-\left(D_{1}-\rho c^{2}\right) & \pm B_{2} \sin \theta \cos \theta \\
\pm B_{2} \sin \theta \cos \theta & -\left(D_{2}-\rho c^{2}\right)
\end{array}\right|=0 .
$$

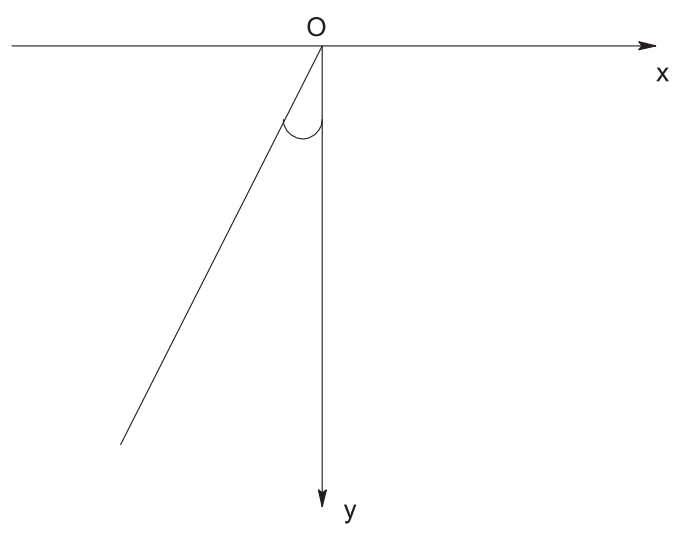

Figure 1. Geometry of the problem. 
The solutions of the quadratic equation (12) in $\rho c^{2}$ are

$$
2 \rho c^{2}(\theta)=\left(D_{1}+D_{2}\right) \pm\left[\left(D_{1}-D_{2}\right)^{2}+4 B_{2}^{2} \sin ^{2} \theta \cos ^{2} \theta\right]^{1 / 2} .
$$

Thus, in this two-dimensional model of the fibre-reinforced anisotropic medium, there are two types of plane waves whose phase velocities depend on the angle of incidence $\theta$. Let $c_{1}(\theta)$ and $c_{2}(\theta)$ be the values of $c$ associated with the upper and the lower signs respectively, in (13). On neglecting reinforcement parameters, $c_{1}$ and $c_{2}$ reduce to the usual velocities of $P$ and $S$ waves in an isotropic material with Lame constants $\lambda, \mu_{T}$ and density $\rho$. We call the waves with velocities $c_{1}$ and $c_{2}$ as quasi- $P$ and quasi- $S$ waves respectively. We next discuss the reflection of these waves at the free boundary $y=0$.

\section{Quasi-P waves incident at the free boundary}

If quasi- $P$ waves are incident at the boundary $y=0$ of the fibre-reinforced anisotropic semiinfinite medium, we get quasi- $P$ and quasi- $S V$ waves as reflected waves. We may, therefore, assume the total displacement to be of the form

$$
\begin{aligned}
& u=U_{0} \exp \left(i R_{1}\right)+U_{1} \exp \left(i S_{1}\right)+U_{2} \exp \left(i S_{2}\right), \\
& v=V_{0} \exp \left(i R_{1}\right)+V_{1} \exp \left(i S_{1}\right)+V_{2} \exp \left(i S_{2}\right),
\end{aligned}
$$

where

$$
\begin{aligned}
R_{1} & =\left(\omega / c_{1}\right)\left[c_{1} t-(x \sin e-y \cos e)\right], \\
S_{1} & =\left(\omega / c_{1}\right)\left[c_{1} t-(x \sin e+y \cos e)\right], \\
S_{2} & =\left(\omega / c_{2}\right)\left[c_{2} t-(x \sin f+y \cos f)\right],
\end{aligned}
$$

are the phase factors associated with the incident quasi- $P$, reflected quasi- $P$ and reflected quasi- $S V$ waves, $e$ being the angle which incident and reflected quasi- $P$ waves make with $y$-axis and $f$ is the angle which the reflected quasi- $S V$ waves make with the $y$-axis. $U_{0}, V_{0} ; U_{1}, V_{1}$; and $U_{2}, V_{2}$ are the amplitude factors associated with the incident quasi- $P$, reflected quasi- $P$ and reflected quasi- $S V$ waves respectively. Since the incident and reflected waves in (14) and (15) must satisfy the equations of motion (5) and (6), we have, as in (9),

$$
\begin{gathered}
-\left[D_{1}(e)-\rho c_{1}^{2}(e)\right] U_{0}+B_{2} \sin e \cos e V_{0}=0, \\
-\left[D_{1}(e)-\rho c_{1}^{2}(e)\right] U_{1}-B_{2} \sin e \cos e V_{1}=0, \\
-\left[D_{1}(f)-\rho c_{2}^{2}(f)\right] U_{2}-B_{2} \sin f \cos f V_{2}=0 .
\end{gathered}
$$

It may be noted that we can obtain another set of similar equations corresponding to (10). But this set will give the same results as the set in (17) due to consistency condition (12). Equations (17) may be written as

$$
U_{0}=\eta_{1} V_{0}, \quad U_{1}=-\eta_{1} V_{1}, \quad U_{2}=-\eta_{2} V_{2},
$$

where

$$
\begin{aligned}
& \eta_{1}=B_{2} \sin e \cos e /\left[D_{1}(e)-\rho c_{1}^{2}(e)\right] \\
& \eta_{2}=B_{2} \sin f \cos f /\left[D_{1}(f)-\rho c_{2}^{2}(f)\right]
\end{aligned}
$$


The total displacement field in (14) and (15) must satisfy the boundary conditions,

$$
\sigma_{21}=\sigma_{22}=0, \quad \text { at } \quad y=0 .
$$

Making use of (2), (14) and (15) in the above boundary conditions, we obtain

$$
\begin{aligned}
& {\left[\left(\cos e / c_{1}\right) U_{0}+\left(-\sin e / c_{1}\right) V_{0}\right] \exp \left[i S_{1}(x, 0)\right]} \\
& \quad+\left[\left(-\cos e / c_{1}\right) U_{1}+\left(-\sin e / c_{1}\right) V_{1}\right] \exp \left[i S_{1}(x, 0)\right] \\
& \quad+\left[\left(-\cos f / c_{2}\right) U_{2}+\left(-\sin f / c_{2}\right) V_{2}\right] \exp \left[i S_{2}(x, 0)\right], \\
& \quad\left[(\lambda+\alpha)\left(-\sin e / c_{1}\right) U_{0}+\left(\lambda+2 \mu_{T}\right)\left(\cos e / c_{1}\right) V_{0}\right] \exp \left[i S_{1}(x, 0)\right] \\
& \quad+\left[(\lambda+\alpha)\left(-\sin e / c_{1}\right) U_{1}+\left(\lambda+2 \mu_{T}\right)\left(-\cos e / c_{1}\right) V_{1}\right] \exp \left[i S_{1}(x, 0)\right] \\
& \quad+\left[(\lambda+\alpha)\left(-\sin f / c_{2}\right) U_{2}+\left(\lambda+2 \mu_{T}\right)\left(-\cos f / c_{2}\right) V_{2}\right] \exp \left[i S_{2}(x, 0)\right]=0,
\end{aligned}
$$

where

$$
c_{1}=c_{1}(e), \quad c_{2}=c_{2}(f),
$$

and where we have used the result

$$
R_{1}(x, 0)=S_{1}(x, 0) .
$$

Since (21) and (22) must be satisfied for all values of $x$, we have

$$
S_{1}(x, 0)=S_{2}(x, 0),
$$

which, on using (16), gives

$$
\sin e / c_{1}(e)=\sin f / c_{2}(f) .
$$

This is the form of Snell's law for the fibre-reinforced anisotropic medium. Equations (21) and (22), with the help of (18) and (25), may be written as

$$
\begin{gathered}
m_{1} V_{0}+m_{1} V_{1}+m_{2} V_{2}=0, \\
n_{1} V_{0}-n_{1} V_{1}+n_{2} V_{2}=0,
\end{gathered}
$$

where

$$
\begin{aligned}
m_{1} & =\left(\eta_{1} \cos e-\sin e\right) / c_{1}, \\
m_{2} & =\left(\eta_{2} \cos f-\sin f\right) / c_{2}, \\
n_{1} & =\left[(\lambda+\alpha) \eta_{1} \sin e-\left(\lambda+2 \mu_{T}\right) \cos e\right] / c_{1}, \\
n_{2} & =-\left[(\lambda+\alpha) \eta_{2} \sin f-\left(\lambda+2 \mu_{T}\right) \cos f\right] / c_{2} .
\end{aligned}
$$

The amplitude ratios for the reflected waves are obtained from (18), (27) and (28) as

$$
\begin{aligned}
& U_{1} / U_{0}=\left(m_{1} n_{2}-m_{2} n_{1}\right) / \Delta, \quad U_{2} / U_{0}=2 \eta_{2} m_{1} n_{1} / \eta_{1} \Delta, \\
& V_{1} / V_{0}=\left(m_{2} n_{1}-m_{1} n_{2}\right) / \Delta, \quad V_{2} / V_{0}=-2 m_{1} n_{1} / \Delta,
\end{aligned}
$$


where

$$
D=m_{1} n_{2}+m_{2} n_{1}
$$

Equation (30) gives the amplitude ratios for the horizontal component of the displacement. Similarly, (31) gives the amplitude ratios for the vertical component. From (30) and (31), we obtain the following expressions for the amplitude ratios for the total displacement for reflected $\mathrm{q} P$ and $\mathrm{q} S V$ waves when $\mathrm{q} P$ waves are incident at the free boundary

$$
\begin{aligned}
& Z_{1}^{P P}=\left|\left(m_{2} n_{1}-m_{1} n_{2}\right) / \Delta\right|, \\
& Z_{2}^{P S}=\left\|2 m_{1} n_{1} / \Delta\right\|\left[\left(\eta_{2}^{2}+1\right) /\left(\eta_{1}^{2}+1\right)\right]^{1 / 2} .
\end{aligned}
$$

\section{Quasi-SV waves incident at the free boundary}

We assume that the incident and reflected quasi- $S V$ waves make an angle $f$ with the $y$-axis and the reflected quasi- $P$ waves make an angle $e$ with this axis. Amplitude ratios in this case may be obtained as in the previous section. We find

$$
\begin{aligned}
& U_{1} / U_{0}=2 \eta_{1} m_{2} n_{2} / \eta_{2} \Delta, \quad U_{2} / U 0=\left(m_{2} n_{1}-m_{1} n_{2}\right) / \Delta, \\
& V_{1} / V_{0}=-2 m_{2} n_{2} / \Delta, \quad V_{2} / V_{0}=\left(m_{1} n_{2}-m_{2} n_{1}\right) / \Delta,
\end{aligned}
$$

where $U_{0}, V_{0}$ are the amplitude factors of the incident quasi- $S V$ waves.

From (35) and (36), we obtain the amplitude ratios for the total displacement for reflected $\mathrm{q} P$ and $\mathrm{q} S V$ waves as

$$
\begin{aligned}
Z_{1}^{S P} & =\left\|2 m_{2} n_{2} / \Delta\right\|\left[\left(\eta_{1}^{2}+1\right) /\left(\eta_{2}^{2}+1\right)\right]^{1 / 2}, \\
Z_{2}^{S S} & =\left|\left(m_{1} n_{2}-m_{2} n_{1}\right) / \Delta\right| .
\end{aligned}
$$

\section{Numerical results and discussion}

To study the effect of reinforcement on wave propagation, we use the following numerical values for the physical constants

$$
\begin{aligned}
\lambda & =7.59 \times 10^{9} \mathrm{~N} / \mathrm{m}^{2}, & \mu_{T} & =1.89 \times 10^{9} \mathrm{~N} / \mathrm{m}^{2}, \\
\mu_{L} & =2.45 \times 10^{9} \mathrm{~N} / \mathrm{m}^{2}, & \alpha & =-1.28 \times 10^{9} \mathrm{~N} / \mathrm{m}^{2}, \\
\beta & =0.32 \times 10^{9} \mathrm{~N} / \mathrm{m}^{2}, & \rho & =7800 \mathrm{~kg} / \mathrm{m}^{3} .
\end{aligned}
$$

Making use of Snell's law given by (26), the angles of reflection for $\mathrm{q} P$ and $\mathrm{q} S V$ waves are computed for various values of the angle of incidence of $\mathrm{q} S V$ and $\mathrm{q} P$ waves respectively. Figure 2 gives the angle of reflection of qSV waves for various values of the angle of incidence of $\mathrm{q} P$ waves with and without reinforcement. For the present choice of the physical constants, the angle of reflection for $\mathrm{q} S V$ waves in the presence of reinforcement increases more rapidly than in the absence of reinforcement. Figure 3 gives the angle of reflection of q $P$ waves for various values of the angle of incidence of $\mathrm{q} S V$ waves with and without reinforcement.

The amplitude ratios for reflected $\mathrm{q} P$ and $\mathrm{q} S V$ waves are computed for incident $\mathrm{q} P$ and $\mathrm{q} S V$ waves. The variations of these amplitude ratios with the angle of incidence are shown graphically in figures 4 and 5 for incident q $P$ and q $S V$ waves, respectively. The dotted lines with and without crosses represent the variations of the amplitude ratios for reflected waves in the absence of reinforcement. The solid lines with and without crosses represent the amplitude ratios with reinforcement. 


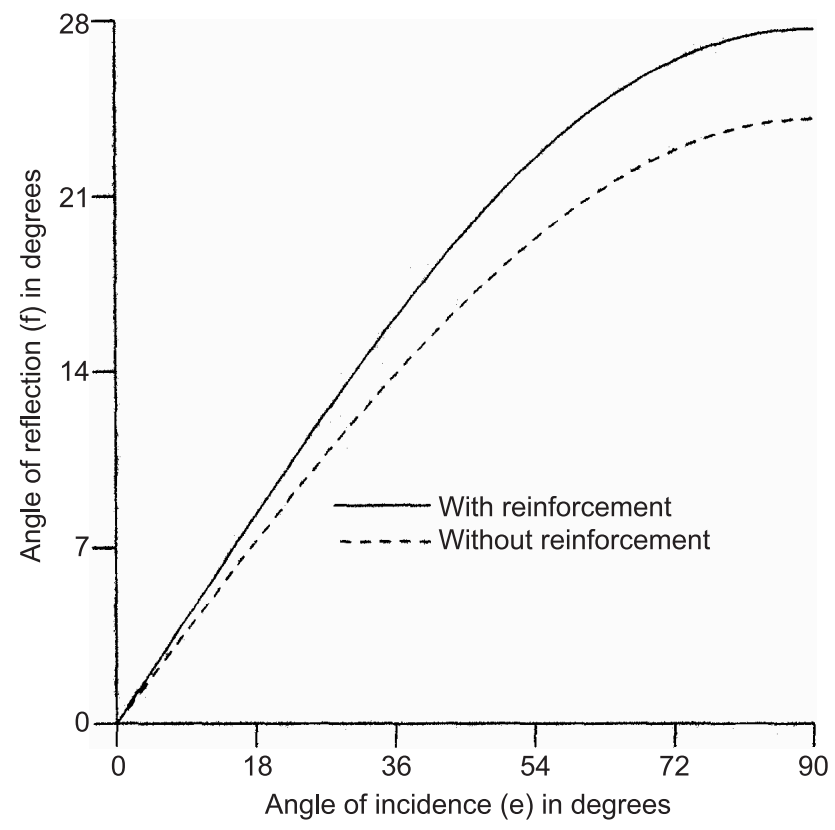

Figure 2. Variation of the angle of reflection of q $S V$ waves with the angle of incidence of $\mathrm{q} P$ waves.

\subsection{Incident quasi-P wave}

The variations of the amplitude ratios for reflected $\mathrm{q} P$ and $\mathrm{q} S V$ waves with the angle of incidence of $\mathrm{q} P$ waves are shown in figure 4 by solid lines with crosses and solid lines, respectively. The amplitude ratio for reflected q $P$ is one at $e=0^{\circ}$ and $e=90^{\circ}$ and attains

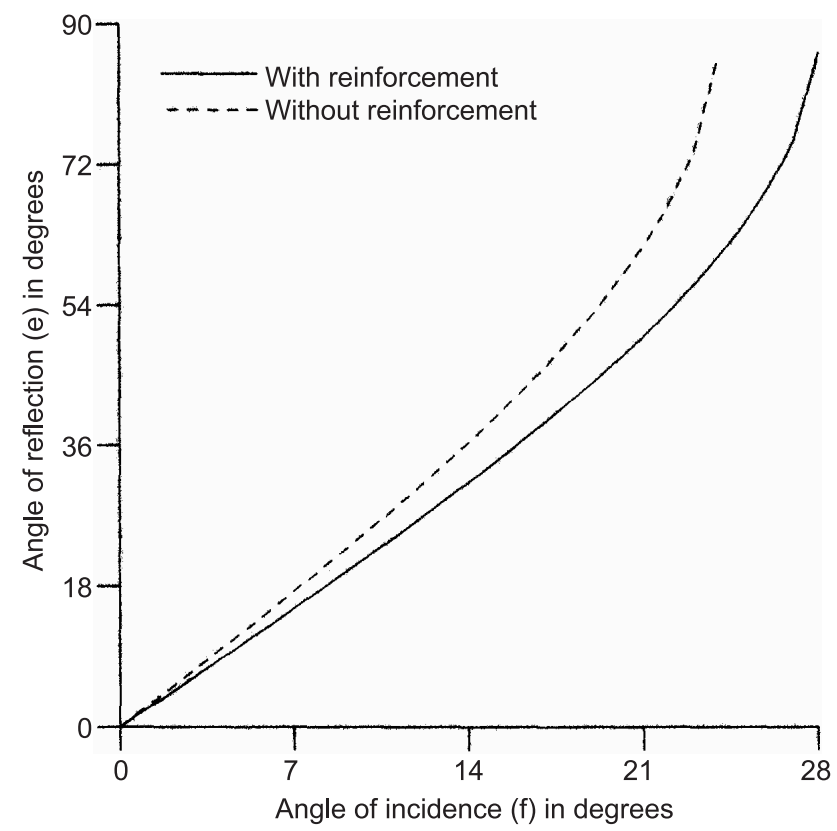

Figure 3. Variation of the angle of reflection of $\mathrm{q} P$ waves with the angle of incidence of $\mathrm{q} S V$ waves. 


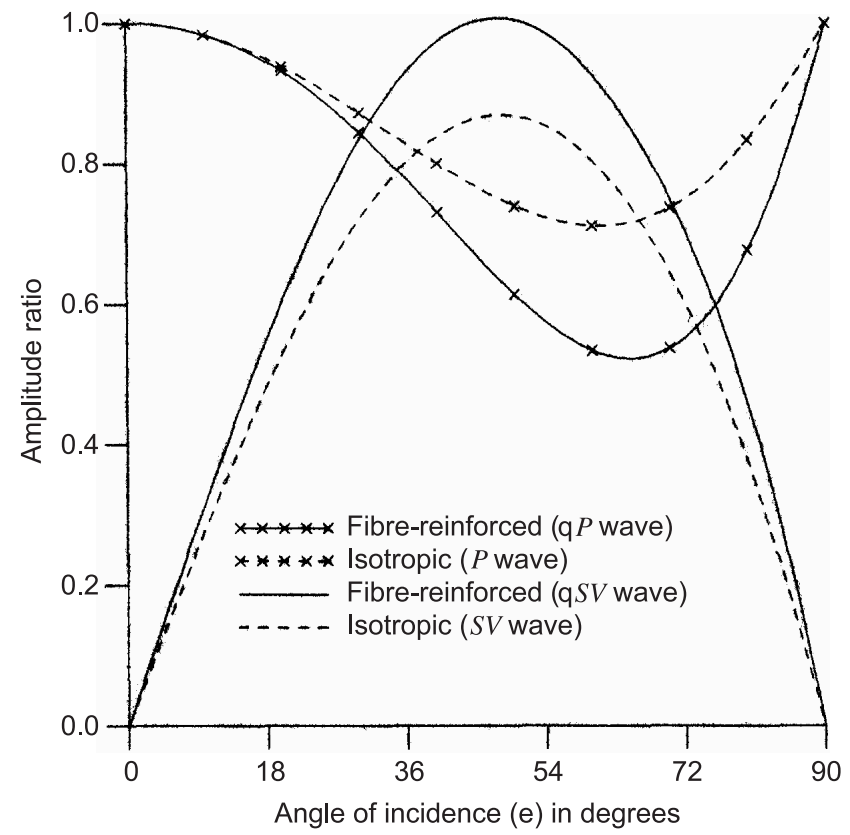

Figure 4. Variation of amplitude ratios for reflected waves with the angle of incidence of $\mathrm{q} P$ waves.

its minimum at $e=65^{\circ}$. Also, the amplitude ratio for reflected $\mathrm{q} S V$ has its value zero at $e=0^{\circ}$ and $e=90^{\circ}$ and attains its maximum at $e=48^{\circ}$. A comparison between solid and dotted lines reveals that the effect of reinforcement on amplitude ratios of reflected $\mathrm{q} P$ and $\mathrm{q} S V$ waves is significant.

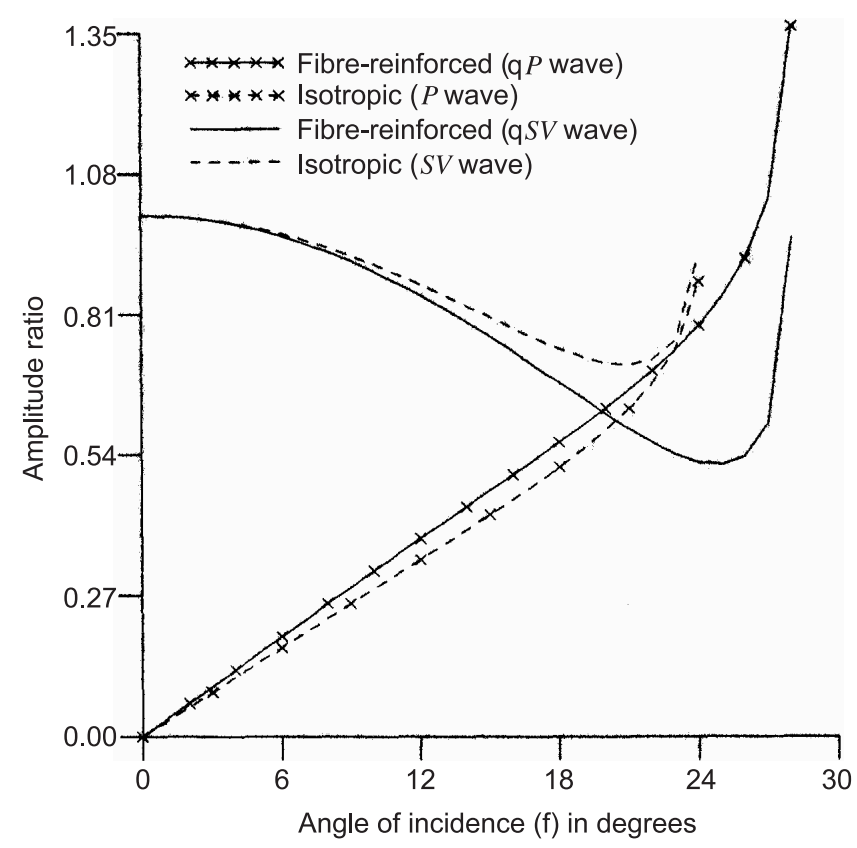

Figure 5. Variation of amplitude ratios for reflected waves with the angle of incidence of q $S V$ waves. 


\subsection{Incident quasi-SV wave}

The variations of the amplitude ratios for reflected $\mathrm{q} P$ and $\mathrm{q} S V$ waves with the angle of incidence of $\mathrm{q} S V$ wave are shown in figure 5 by solid lines with crosses and solid lines respectively. The amplitude ratio for reflected $\mathrm{q} P$ is zero at $f=0^{\circ}$ and it attains its maximum near $f=28^{\circ}$. Beyond $f=28^{\circ}$, it is zero for all angles of incidence. Thus the critical angle for reflected $\mathrm{q} P$ waves is $28^{\circ}$. Also, the amplitude ratio for reflected $\mathrm{q} S V$ wave is one at $f=0^{\circ}$ and decreases to its minimum at $f=25^{\circ}$. For the range $25^{\circ} \leq f \leq 28^{\circ}$, it increases. Beyond $f=28^{\circ}$, it is one for all angles of incidence. The effect of reinforcement as defined by the physical constants chosen is to increase the critical angle from about $24^{\circ}$ to about $28^{\circ}$.

\section{Conclusions}

Equations (33), (34), (37) and (38) give the amplitude ratios of the reflected waves when $\mathrm{q} P$ and $\mathrm{q} S V$ waves are incident at the free surface of a fibre-reinforced, homogeneous, anisotropic, elastic half-space. It is assumed that the plane of incidence contains the reinforcement direction. We have verified that, on putting the reinforcement parameters $\alpha, \beta$ and $\left(\mu_{L}-\mu_{T}\right)=0$ each, the amplitude ratios obtained in the present study coincide with the amplitude ratios for an isotropic half-space as given by Ben-Menahem \& Singh (1981, pp 93-95). We have found that reinforcement has a significant effect on the amplitude ratios and alters the critical angle considerably.

The authors thank the Council of Scientific and Industrial Research, New Delhi for financial support to SJS.

\section{References}

Belfield A J, Rogers T G, Spencer A J M 1983 Stress in elastic plates reinforced by fibres lying in concentric circles. J. Mech. Phys. Solids 31: 25-54

Ben-Menahem A, Singh S J 1981 Seismic waves and sources (New York: Springer-Verlag)

Ditri J J, Rose J L 1992 On the reflection of plane waves in transversely isotropic media. J. Acoust. Soc. Am. 92 : 3003-3006

Hashin Z, Rosen W B 1964 The elastic moduli of fibre reinforced materials. J. Appl. Mech. 31: 223232

Sengupta P R, Nath S 2001 Surface waves in fibre-reinforced anisotropic elastic media. Sādhanā 26: 363-370

Singh S J 2002 Comments on "Surface waves in fibre-reinforced anisotropic elastic media," by Sengupta and Nath [Sädhanā 26: 363-370 (2001)]. Sädhanā 27: 1-3

Singh S J, Khurana S 2002 Reflection of P and SV waves at the free surface of a monoclinic elastic half-space. Proc. Indian Acad. Sci. (Earth Planet. Sci.) 111: 401-412

Zilmer M, Gajewski D, Kashtan B M 1997 Reflection coefficients for weak anisotropic media. Geophys. J. Int. 132: 159-166 